\title{
Dupla tarefa e mobilidade funcional de idosos ativos
}

\author{
Dual task and functional mobility of active elderly
}

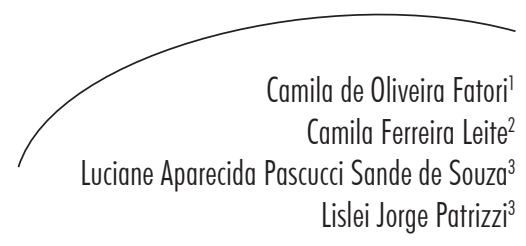

Resumo

Introdução: Define-se "dupla tarefa" como a capacidade de efetivar uma ação primordial incorporada a uma segunda atividade. Em idosos, é presumível que a execução de ações simultâneas comprometa o equilíbrio postural, favorecendo quedas nesta população. Objetivos: Avaliar o efeito de duplas tarefas na mobilidade funcional de idosos ativos e correlacionar o tempo utilizado para sua realização com a idade do indivíduo. Métodos: Estudo transversal e observacional, que utiliza amostra de conveniência. Dezenove idosos do Programa Universidade Aberta à Terceira Idade da Universidade Federal do Triângulo Mineiro, com idade entre 60 e 87 anos, classificados como ativos pelo International Physical Activity Questionnaire (IPAQ) e com escore mínimo de 23 pontos no Miniexame do Estado Mental (MEEM) foram avaliados em nove testes sequenciais. $O$ primeiro deles exigia uma única tarefa, representada pelo teste Timed Up \& Go (TUG), utilizado para avaliação da mobilidade funcional de idosos. O TUG foi repetido em todos os demais testes, que incorporaram uma segunda ação - atividade manual em dois testes e atividade cognitiva em seis testes. Resultados: Foram observados valores maiores em relação ao tempo gasto pelos idosos na realização de tarefas associadas, tanto motoras quanto motora-cognitivas, quando comparadas com a tarefa simples. Correlação positiva entre idade e os testes que incorporam atividade cognitiva à realização do TUG foi estabelecida. Conclusão: Idosos ativos necessitam de maior tempo para a realização da dupla tarefa motora envolvendo funções distintas e coordenadas entre os membros superiores, o mesmo ocorrendo para a execução das duplas tarefas motora-cognitivas. Quanto maior a idade, maior o tempo para realização de duplas tarefas, principalmente quando associadas a atividades que exijam memória de curto prazo.

\footnotetext{
Universidade Federal do Triângulo Mineiro, Curso de Fisioterapia. Uberaba, MG, Brasil.

2 Universidade Federal do Triângulo Mineiro, Programa de Pós-graduação em Ciências da Saúde. Uberaba, MG, Brasil.

3 Universidade Federal do Triângulo Mineiro, Departamento de Fisioterapia Aplicada, Instituto de Ciências da Saúde. Uberaba, MG, Brasil.
}

Palavras-chaves:

Envelhecimento. Acidentes por Quedas. Tarefa de Controle Duplo. Idoso. 


\section{Abstract}

Introduction: Dual task can be defined as the ability to perform a primary action embedded in a second activity. In the elderly, it is presumed that the performance of concurrent actions disturb the postural balance, favoring falls in this population. Aims: To evaluate the effect of dual tasks in functional mobility of active seniors and correlate the time used for its realization with the individual's age. Methods: Cross-sectional, observational study with a convenience sample. Nineteen elderly from Universidade Aberta à Terceira Idade, Universidade Federal do Triângulo Mineiro, Minas Gerais state, Brazil, aged between 60 and 87 years, classified as physically active by the International Physical Activity Questionnaire (IPAQ) and with a minimum score that reaches 23 points on the Mini-Mental State Examination (MMSE) were evaluated during 9 sequential tests. The first one required a single task, which was represented by the Timed Up \& Go (TUG) test, used to assess functional mobility of seniors. TUG was repeated in all other tests, in which was included a second action - manual activity in two tests and cognitive activity in six tests. Results: The elderly spent longer period performing associated tasks considering both motor and motor-cognitive combined actions when compared with time spent during the simple task. Positive correlation between age and the tests that incorporate cognitive activity to achieve the TUG was established. Conclusion: Physically active seniors require a longer period to perform the dual motor task involving distinct functions and coordinates between the upper limbs, and the same occurs for the performance of motor-cognitive dual tasks. The higher the age, the greater the time to perform double tasks, especially when associated with activities requiring short-term memory.

\section{INTRODUÇ̃̃O}

A dupla tarefa pode ser definida como o ato de realizar uma atividade primária, para a qual é destinado o maior foco da atenção, incorporada a uma segunda atividade executada ao mesmo tempo. ${ }^{1}$ A realização de duas tarefas simultâneas é comum no $\operatorname{cotidiano}^{2,3}$ e representa uma capacidade altamente vantajosa para o indivíduo, podendo ser considerada um pré-requisito para uma vida normal. ${ }^{4}$

A duplicidade de tarefas, sendo elas motoras ou cognitivas, ocorre em nível cortical, propiciando que uma intervenha na outra. ${ }^{5}$ Assim sendo, apesar da facilidade com que alguns atos simultâneos possam ser rotineiramente executados, a integralidade de sua ação demanda um alto processamento neural. ${ }^{2}$ Em alguns casos, a realização de dupla tarefa pode ser prejudicada quando as ações exigidas excedem a capacidade do sistema cognitivo ${ }^{3}$ ou ainda quando resultam no envolvimento simultâneo de circuitos neurais específicos às duas ações. ${ }^{6}$

\author{
Key words: Aging. \\ Accidental Falls. Dual Task. \\ Elderly.
}

Em idosos, a busca pela concentração focal ao executar ações simultâneas compromete o equilíbrio postural, podendo causar diminuição da autonomia coletiva, alterações em atividades corriqueiras, depressão, declínio da mobilidade, medo e apreensão, devido a maiores riscos de quedas, com consequente dependência e maiores gastos com tratamento de saúde. ${ }^{7}$ As alterações na mobilidade comprometem a realização de suas atividades de vida diária, ${ }^{8}$ além de aumentarem os riscos de quedas, ${ }^{9}$ comprometendo de maneira inevitável a qualidade de vida dessa população.

Um teste frequentemente utilizado para avaliar o equilíbrio e a mobilidade funcional de idosos é o Timed Up \& Go (TUG). ${ }^{10,11}$ Shumway-Cook et al. ${ }^{12}$ mostram que, ao realizar o TUG, os idosos que não apresentam alterações de equilíbrio nem dependências físicas executam o teste em 10 segundos ou menos. Aqueles independentes nas transferências básicas efetuam o teste em 20 segundos ou menos, e os que precisam de mais de 20 segundos para concluir o teste apresentam mobilidade prejudicada e dependência em 
atividades de vida diária. ${ }^{11}$ Pode-se considerar que idosos que executam o TUG em um tempo maior que 14 segundos apresentam alto risco de quedas. ${ }^{11,12}$

Alguns estudos sugerem que a avaliação do equilíbrio e da mobilidade funcional seja realizada durante as duplas tarefas, uma vez que as quedas geralmente acontecem quando duas ou mais tarefas se associam. ${ }^{5,13-15}$ A relevância de se identificar o desempenho funcional de idosos durante a execução de duas atividades simultâneas considera o maior risco de quedas que esta população apresenta ao executar dupla tarefa, quando comparado à execução de um ato único. Ademais, informações acerca da mobilidade funcional de idosos podem ser valiosas para predizer quedas, e úteis para orientar programas preventivos. ${ }^{16}$

Assim sendo, objetivou-se neste estudo avaliar o efeito de duplas tarefas na mobilidade funcional de idosos ativos e correlacioná-lo com a idade.

\section{METODOLOGIA}

Trata-se de estudo transversal e observacional, tendo uma amostra de conveniência, constituída de idosos ativos e pertencentes ao programa Universidade Aberta à Terceira Idade da Universidade Federal do Triângulo Mineiro (UATI/UFTM), realizado no período de junho e julho de 2013. O estudo foi aprovado pelo Comitê de Ética em Pesquisa da UFTM, sob protocolo $n^{\circ} 2.596 / 2013$. Todos os participantes assinaram o Termo de Consentimento Livre e Esclarecido.

Participaram do estudo 19 idosos de ambos os gêneros, com idade superior a 60 anos, alfabetizados, eutróficos e que aceitaram participar da pesquisa. Foram adotados como critérios de exclusão: (a) idosos classificados como sedentários por meio do International Physical Activity Questionnaire (IPAQ); (b) idosos que apresentavam limitação funcional para a marcha (dor incapacitante e/ou sequelas de doenças neurológicas ou ortopédicas), identificada durante avaliação estruturada; (c) idosos que não atingiram a pontuação mínima de 23 pontos estabelecida no Miniexame do Estado Mental (MEEM).

Os participantes foram classificados como ativos ou sedentários por meio do IPAQ, questionário composto por perguntas relacionadas a frequência e duração de caminhadas, atividades cotidianas que exigem esforços físicos de intensidades moderada e vigorosa, além do tempo despendido em atividades executadas em posição sentada. O entrevistado considera para suas respostas o intervalo de tempo de uma semana, tendo como referência uma semana típica ou a última semana. ${ }^{17,18}$ Utilizou-se a versão curta semanal, aplicada na forma de entrevista individual, incluindo, quando necessário, exemplos de atividades que são comuns às pessoas desse grupo etário. ${ }^{18}$ Com base no resultado obtido com o IPAQ, os idosos sedentários ou irregularmente ativos foram excluídos da amostra.

O MEEM, também utilizado na seleção dos participantes, é uma avaliação clínica prática que identifica mudanças do estado cognitivo em pacientes geriátricos. Examina orientação temporal e espacial, memória de curto prazo e evocação, cálculo, praxia e habilidades de linguagem e viso-espaciais. ${ }^{19} \mathrm{O}$ escore do MEEM pode variar de um mínimo de 0 ponto, o qual indica o maior grau de comprometimento cognitivo, até um total máximo de 30 pontos, que corresponde à melhor capacidade cognitiva do individuo. ${ }^{19,20} \mathrm{O}$ valor adotado como ponto de corte mínimo necessário para inclusão dos participantes no estudo foi de 23 pontos. ${ }^{19}$

O risco de quedas foi avaliado por meio de nove testes denominados TUG, TUG manual 1 e 2 e TUG cognitivo 1 a 6 , os quais se encontram descritos a seguir:

- TUG: foi solicitado ao participante levantar de uma cadeira com altura 
de $42 \mathrm{~cm}$, caminhar três metros, girar $180^{\circ}$, voltar e sentar-se novamente. Os participantes foram orientados a utilizar um sapato de uso habitual e percorrer o trajeto caminhando no menor tempo possível.

- TUG manual 1: solicitou-se aos participantes que simultaneamente à execução do TUG, associassem a ação de transferência de moedas entre dois bolsos. Assim sendo, durante todo o trajeto, os voluntários transferiram 10 moedas de 50 centavos de real do bolso direito para o esquerdo. ${ }^{5}$ Foram confeccionados aventais de dois tamanhos (50x61cm e $42 \times 54 \mathrm{~cm}$ ) contendo bolsos, visando a adequação aos diferentes tamanhos corporais dos voluntários.

- TUG manual 2: durante a execução do TUG, foi orientado ao voluntário segurar um copo plástico rígido, em formato cilíndrico, com $10 \mathrm{~cm}$ de altura, contendo água até $5,0 \mathrm{~cm}$ de sua capacidade total. ${ }^{5}$

- TUG cognitivo 1: durante a execução do TUG, os voluntários foram estimulados a repetir a frase "Praticar atividade física faz bem para o corpo e mente". ${ }^{5}$

- TUG cognitivo 2: os voluntários foram orientados a soletrar de trás para frente os dias da semana (de domingo a segundafeira), por todo o trajeto percorrido na realização do TUG. ${ }^{5}$

- TUG cognitivo 3: os voluntários foram orientados a soletrar em ordem decrescente os números de 10 a 1 enquanto concluíam o TUG. ${ }^{21}$

- TUG cognitivo 4: os idosos voluntários foram orientados a memorizar e falar as figuras: tartaruga, bicicleta, árvore, cachorro, igreja e telefone, impressas em papel A4, ocupando a folha toda e mostradas no início do teste durante a execução do TUG. ${ }^{22}$

- TUG cognitivo 5: os voluntários foram orientados a repetir cinco palavras - pipa, rua, menino, boneca e menina - e, ao mesmo tempo, realizar o TUG. ${ }^{22}$

- TUG cognitivo 6: simultaneamente à realização do TUG, os voluntários repetiram cinco números previamente informados (17, 4, 8, 11 e 20).

Para todos os nove testes realizados, sempre após o comando "vai" do avaliador, o cronômetro foi disparado, registrando-se, ao final, o tempo gasto para cada execução das atividades. Para todos os participantes, a ordem da aplicação dos testes foi a mesma: TUG, TUG manual 1 e 2 seguidos dos TUGs cognitivos 1 a 6 , sendo que todos os testes foram aplicados por um único avaliador.

$\mathrm{Na}$ avaliação estatística, realizou-se análise descritiva para a caracterização da amostra, sendo os dados expressos em média e desviopadrão. O teste de Shapiro-Wilk foi utilizado para verificar a normalidade na distribuição dos dados. Para análise intergrupos, foi utilizado o teste ANOVA One-way, seguido do pós-teste de Tukey; e para a correlação dos dados, o teste de correlação de Pearson. A correlação de Pearson foi interpretada como: perfeita positiva $(\mathrm{r}=1)$, forte positiva $(0,8 \leq \mathrm{r}<1)$, moderada positiva $(0,5 \leq \mathrm{r}<0,8)$, fraca positiva $(0,1 \leq \mathrm{r}<0,5)$, ínfima positiva $(0<\mathrm{r}<0,1)$, nula $(=0)$, ínfima negativa $(-0,1<\mathrm{r}<0)$, fraca negativa $(-0,5<\mathrm{r} \leq-0,1)$, moderada negativa $(-0,8<\mathrm{r} \leq-0,5)$, forte negativa $(-1<\mathrm{r} \leq-0,8)$, perfeita negativa $(r=-1)$. Os resultados foram considerados significativos quando $\mathrm{p}<0,05$. $\mathrm{O}$ programa estatístico utilizado foi o MINITAB versão 14.0. 


\section{RESULTADOS}

O presente estudo contou com a participação de 19 indivíduos idosos, sendo 26,31\% do sexo masculino $(n=5)$ e $73,69 \%$ do sexo feminino $(\mathrm{n}=14)$. A média de idade encontrada foi de $69,79( \pm 6,9)$ anos.
Foram observados valores maiores em relação ao tempo gasto pelos idosos na realização de tarefas associadas, tanto motoras quanto cognitivas, quando comparadas com a tarefa simples (TUG). Foi observado maior tempo para a realização das tarefas cognitivo motoras que exigiam memorização (TUG 1,4,5 e 6), segundo a tabela 1 .

Tabela 1. Médias e desvio-padrão do tempo gasto para realização dos testes TUG, TUG motor 1 e 2, TUG cognitivo 1 a 6. Uberaba-MG, 2013.

\begin{tabular}{cccc}
\hline Variável & Média & Intervalo de confiança & p-valor \\
\hline TUG & $9,4 \pm 2$ & $8,4-10,4$ & -- \\
TUG manual 1 & $27,5 \pm 10$ & $22,4-32,7$ & $<0,001$ \\
TUG manual 2 & $11 \pm 3,8$ & $9,2-12,9$ & 0,11 \\
TUG cognitivo 1 & $15,9 \pm 15,3$ & $8,5-23,3$ & $<0,001$ \\
TUG cognitivo 2 & $12,1 \pm 6,3$ & $9,0-15,2$ & 0,08 \\
TUG cognitivo 3 & $12,8 \pm 4$ & $10,8-14,7$ & 0,003 \\
TUG cognitivo 4 & $20 \pm 10,5$ & $14,9-25$ & $<0,001$ \\
TUG cognitivo 5 & $15,5 \pm 8,9$ & $11,2-19,8$ & $<0,001$ \\
TUG cognitivo 6 & $16,2 \pm 9,9$ & $11,4-20,9$ & $<0,001$ \\
\hline
\end{tabular}

Os valores de p referem-se às comparações realizadas entre o teste TUG com os demais. 
Os resultados da pesquisa mostram correlação positiva entre idade e os testes que incorporam atividade cognitiva à realização do TUG (TUGs cognitivos 1, 2, 3 e 5), como pode ser observado na tabela 2.

Tabela 2. Correlação entre idade e os TUGs realizados. Uberaba-MG, 2013.

\begin{tabular}{ccc}
\hline & Idade & Correlação \\
\hline TUG & $\mathrm{r}=0,068$ & Ínfima positiva \\
& $\mathrm{p}=0,782$ & \\
$\mathrm{r}=0,054$ & Ínfima positiva \\
$\mathrm{p}=0,828$ & \\
TUG manual 1 & $\mathrm{r}=-0,073$ & Ínfima negativa \\
TUG cognitivo 1 & $\mathrm{p}=0,767$ & \\
& $\mathrm{r}=0,487$ & Fraca positiva \\
TUG cognitivo 2 & $\mathrm{p}=0,034^{*}$ & \\
& $\mathrm{r}=0,469$ & Fraca positiva \\
TUG cognitivo 3 & $\mathrm{p}=0,043^{*}$ & Moderada positiva \\
TUG cognitivo 4 & $\mathrm{r}=0,551$ & \\
TUG cognitivo 5 & $\mathrm{p}=0,015^{*}$ & Fraca negativa \\
TUG cognitivo 6 & $\mathrm{r}=-0,399$ & Moderada positiva \\
& $\mathrm{p}=0,091$ & \\
& $\mathrm{r}=0,587$ & Fraca positiva \\
\hline
\end{tabular}

*significância estatística.

\section{DISCUSSÃO}

A dupla tarefa é essencial para a realização de atividades básicas e instrumentais de vida diária; no entanto, estudos mostram que a associação de duas ou mais tarefas pode afetar o equilíbrio, promovendo maiores índices de quedas. ${ }^{4,23-25} \mathrm{Um}$ dos objetivos deste estudo foi avaliar o efeito das duplas tarefas na mobilidade funcional de idosos ativos. "Mobilidade funcional" é um termo que se refere ao equilíbrio e marcha, atividades comuns no dia a dia. ${ }^{26}$ Esta mobilidade está relacionada diretamente com a capacidade funcional, podendo ser avaliada por meio do tempo utilizado para completar o percurso proposto pelo TUG.
No presente estudo, observou-se a necessidade de maior tempo para a realização das duplas tarefas propostas, quando comparadas com o tempo gasto para a realização do TUG convencional, exceto para a dupla tarefa motora - TUG manual 2 - e para a dupla tarefa cognitivo motora - TUG cognitivo 2 (tabela 1).

O TUG manual 1 exigiu maior tempo de todos os participantes para a realização da tarefa proposta. Este achado se justifica pelo fato de que altas demandas de processamento para a execução de duplas tarefas são particularmente verdadeiras quando funções qualitativamente distintas são coordenadas entre os membros superiores. ${ }^{2}$ 
Mesmo também exigindo uma atividade manual com membro superior, o TUG manual 2 exigiu dos participantes controle motor de uma única mão para segurar o copo, sendo que a outra mão não realizou qualquer atividade simultânea. Este achado chama a atenção para a associação de tarefas diversificadas, durante o treinamento e reabilitação física de idosos. Ademais, esta parece ser uma atividade comum ao cotidiano, que foi refletida na facilidade dos voluntários ao executá-la.

O tempo utilizado para a realização das tarefas é inversamente proporcional ao nível de capacidade funcional. ${ }^{11}$ No entanto, este fato não pode ser considerado um indicador mais sensível para as quedas quando comparado com o TUG convencional. ${ }^{12} \mathrm{O}$ tempo utilizado para a realização das duplas tarefas cognitivo-motoras (TUG cognitivo 1, 3, 4, 5 e 6) indicam maior interferência na mobilidade e na capacidade funcional. Uma exceção a este achado foi observada na realização da dupla tarefa cognitivo-motora - TUG 2, quando se solicitou ao participante soletrar os dias da semana de forma associada a tarefa motora (TUG convencional). Possivelmente, esse resultado se justifica pelo fato de que a tarefa cognitiva exigida foi independente de qualquer processo de memorização. A repetição das palavras que rotineiramente são utilizadas pelos idosos e que, por essência, obedecem a uma sequência ao oferecerem referências temporais, resultou em menor dificuldade quando comparada às outras atividades cognitivas realizadas.

Segundo Moraes, ${ }^{27}$ com o envelhecimento ocorre uma lentificação nos processos cognitivos, o que dificulta a realização simultânea de várias tarefas e retarda a aprendizagem e o resgate das informações. Estudos comprovam que inúmeras vezes o déficit de memória dificulta a realização das atividades de vida diária, causando dependência e ausência de autonomia. ${ }^{28,29}$ Houve correlação positiva entre idade e os TUGs cognitivos 1, 2, 3 e 5, ressaltando-se que as atividades associadas ao TUG nestes quatro testes dependiam de memorização, sendo o déficit de memória a principal queixa dos participantes ao executá-los.

Outro objetivo do estudo foi correlacionar a idade com o tempo utilizado para a realização do TUG, TUG manual 1 e 2 e TUG cognitivo 1 a 6 . Os resultados mostram que, quanto maior a idade, maior o tempo para a realização das duplas tarefas cognitivo-motoras. Relata-se que a eficácia ao realizar tarefas motoras comprometese com o envelhecimento, ${ }^{30}$ comprovando que o tempo é influenciado pela idade durante a execução das duplas tarefas. Bohanon ${ }^{31}$ demonstrou que, para a realização do TUG, idosos com idade entre 60 e 69 necessitam de 8,1 segundos; dos 70 aos 79 anos, 9,2 segundos; e dos 80 aos 99 anos, 11,3 segundos.

Algumas limitações do estudo foram o número reduzido de participantes e a restrição do estudo à amostra de idosos ativos. Portanto, sugere-se que outras pesquisas com amostra maior sejam realizadas, comparando-se os achados aos obtidos por idosos sedentários, institucionalizados e até jovens ativos. Ademais, ao que é de conhecimento dos autores inexiste na literatura um classificador específico para interpretação aprofundada dos achados temporais obtidos a partir da realização das duplas tarefas realizadas.

\section{CONCLUSÃ̃O}

O estudo aponta que idosos ativos necessitam de maior tempo para a realização da dupla tarefa motora, desde que esta envolva funções distintas e coordenadas entre os membros superiores, o mesmo ocorrendo para a execução das duplas tarefas cognitivomotoras. Considerando a influência etária, conclui-se que quanto maior a idade, maior o tempo para realização de duplas tarefas, principalmente quando associadas a atividades que exijam memória de curto prazo. 


\section{REFERÊNCIAS}

1. O’Shea S, Morris ME, Iansek R. Dual task interference during gait in people with Parkinson disease: effects of motor versus cognitive secondary tasks. Phys Ther 2002;82(9):888-97.

2. Serrien DJ, Pogosyan AH, Brown P. Cortico-cortical coupling patterns during dual task performance. Exp Brain Res 2004;157(1):79-84. Epub 2004 Feb 17.

3. Bowen A, Wenman R, Mickelborough J, Foster J, Hill E, Tallis R. Dual-task effects of talking while walking on velocity and balance following a stroke. Age Ageing 2001;30(4):319-23.

4. Teixeira NB, Alouche SR. O desempenho da dupla tarefa na doença de Parkinson. Rev Bras Fisioter 2007;11(2):127-32.

5. Barbosa JMM, Prates BSS, Gonçalves CF, Aquino AR, Parentoni AN. Efeito da realização simultânea de tarefas cognitivas e motoras no desempenho funcional de idosos da comunidade. Fisioter Pesqui 2008;15(4):374-9.

6. Johannsen L, Li KZ, Chechlacz M, Bibi A, Kourtzi Z, Wing AM. Functional neuroimaging of the interference between working memory and the control of periodic ankle movement timing. Neuropsychologia 2013;51(11):2142-53.

7. Gomes CD, Mendes AG, Da Silva CF, De Albuquerque CE. Análise do equilíbrio postural associado a dupla tarefa em idosos saudáveis. FIEP Bulletin On-line 2012;82.

8. Begate PS, Ricardo ACM , Sawazki G. Avaliação do desempenho funcional de idosos institucionalizados e não Institucionalizados através do teste de mobilidade timed up and go (TUG). Rev Funcional 2009;2(2):43-52.

9. Ferratin AC, Borges CF, Morelli JGS, Rebelatto JR. A Execução de AVDS e mobilidade funcional em idosos institucionalizados e não-institucionalizados. Fisioter Mov 2007;20(3):115-21.

10. Ayan C, Cancela JM, Gutiérrez A, Prieto I. Influence of the cognitive impairment level on the performance of the Timed "Up \& Go" Test (TUG) in elderly institutionalized people. Arch Gerontol Geriatr 2013;56(1):44-9.

11. Alvarenga PP, Pereira DS, Anjos DMC. Mobilidade funcional e função executiva em idosos diabéticos e não diabéticos. Rev Bras Fisioter 2010;14(6):491-6.

12. Shumway-Cook A, Brauer S, Woollacott M. Predicting the probability for falls in communitydwelling older adults using the Timed Up \& Go Test. Phys Ther 2001;80(9):896-90.
13. Bloem BR, Valkenburg VV, Slabbekoorn M, Willemsen MD. The multiple tasks test: development and normal strategies. Gait Posture 2001;14(3): 191-202.

14. Melzer I, Benjuya N, Kaplanky J. Age-related changes of postural control: effect of cognitive tasks. Gerontology 2001;47(4):189-94.

15. Hauer K, Marburguer C, Oester P. Motor performance deteriorates with simultaneously performed cognitive tasks in geriatric patients. Arch Phys Med Rehabil 2002;83(2):217-23.

16. Thrane G, Joakimsen RM, Thornquist E. The association between timed up and go test and history of falls: the Tromsø study. BMC Geriatr 2007;7:1-7.

17. Pardini R, Matsudo S, Araújo T, Matsudo V, Andrade E, Braggion $\mathrm{G}$, et al. Validação do questionário internacional de nível de atividade física (IPAQ versão 6): estudo piloto em adultos jovens brasileiros. Rev Bras Ciênc Mov 2001;9(3):45-51.

18. Benedetti TRB, Borges LJ, Petroski EL, Gonçalves LHT. Atividade física e estado de saúde mental de idosos. Rev Saúde Pública 2008;42(2):302-7.

19. Lourenço RA, Veras RP. Mini-Exame do Estado Mental: características psicométricas em idosos ambulatoriais. Rev Saúde Pública 2006;40(4):712-9.

20. Valle EA, Castro-Costa E, Firmo JOA, Uchoa E, Lima-Costa MF. Estudo de base populacional dos fatores associados ao desempenho no Mini Exame do Estado Mental entre idosos: Projeto Bambuí. Cad Saúde Pública 2009;25(4):918-26.

21. Andrade LP. Funções cognitivas frontais e controle postural na doença de Alzheimer: efeitos do Programa de Intervenção Motora com Tarefa Dupla [dissertação]. Rio Claro, SP: Universidade Estadual Paulista; 2013.

22. Lemos PV. Análise do controle postural de idosas saudáveis [dissertação]. São Paulo: Universidade Cidade de São Paulo; 2009.

23. Custódio EB, Malaquias J Júnior, Voos MC. Relação entre cognição (função executiva e percepção espacial) e equilíbrio de idosos de baixa escolaridade. Fisioter Pesqui 2010;17(1):46-51.

24. Teixeira PPS, Voos MC, Machado MSA, Castelli LZ, Do Valle LER, Piemonte MEP. Interferência mútua entre atividade visual e atividade motora em jovens e idosos. Fisioter Pesqui 2008;1(2):142-8.

25. Gehring PR, Bertolassi MA, Nunes MES, Basso L, Meira CM Júnior, Dos Santos AS. Desempenho de 
idosos em uma tarefa motora de demanda dupla de controle. Rev Bras Educ Fís Esp 2009;23(3):211-20.

26. Podsiadlo D, Richardson S. The timed "Up \& Go": a test of basic functional mobility for frail elderly persons. J Am Geriatr Soc 1991;39(2):142-8.

27. Moraes EN. Atenção à saúde do idoso: aspectos conceituais. Brasília: Organização Pan-Americana da saúde; 2012. p. 14-30.

28. Almeida MHM, Beger MLM, Watanabe HAW. Oficina de memória para idosos: estratégia para promoção da saúde. Interface Comun Saúde Educ 2007;11(22):271-80.
29. Olchik, MR et al. Revisão: efeitos do treino de memória em idosos normais e em idosos com comprometimento cognitivo leve. TEMAS EM PSICOLOGIA. Ribeirão Preto. v. 20, n.1, p. 235-245 de 2012.

30. Andrade LP, Mattos D, Maineri NS, Farina J, Oliveira A, Yassuda MS. Efeitos de tarefas cognitivas no controle postural de idosos: uma revisão sistemática. Motricidade 2011;7(3):19-28.

31. Bohanon RW. Reference values for the Timed Up and Go test: a descriptive meta-analysis. J Geriatr Phys Ther 2006;29(2):64-8. 